\title{
Développement d'un générateur de type Blumlein pour des sources EUV à décharges capillaires rapides
}

\author{
S. Götze, D. Hong, R. Dussart, C. Cachoncinlle, J. Pons, J.-M. Pouvesle, \\ C. Fleurier et R. Viladrosa
}

GREMI-ESPEO, Université d'Orléans, 14 rue d'Issoudun, BP. 6744, 45067 Orléans cedex 2, France

Résumé: A la suite des différents travaux qui ont démontré l'efficacité des lasers X pompés par décharge capillaire, plusieurs schémas de ligne de mise en forme d'impulsion ont été proposés pour injecter dans ces plasmas des densités de puissance considérables. Au GREMI nous avons développé un générateur d'impulsion permettant de produire un plasma fortement émissif dans le domaine spectral de l'EUV à partir d'une décharge capillaire rapide de type Z-pinch.

\section{INTRODUCTION}

Le développement de sources extrêmement brillantes dans le domaine de l'ultraviolet lointain semble être depuis quelques années le défi de la micro-électronique des années à venir. Actuellement le cahier des charges est tel qu'aucune des sources EUV existantes, cohérentes et incohérentes, ne le satisfait pleinement : l'encombrement et le coût des synchrotrons semblent peu compatibles avec le tissu industriel actuel, les sources plasma créées par impact laser sur jet, plus compactes, n'offrent pas encore suffisamment de flux de photons, et, enfin, les sources par décharge électrique dans un capillaire, si elles sont d'une efficacité énergétique incomparable, présentent actuellement l'inconvénient de projeter des débris susceptibles de limiter la durée de vie des dépôts sur les optiques EUV.

Au GREMI nous avons depuis quelques années développé différentes sources par décharge capillaire [1]. Nous présentons ici les performances d'une source EUV construite autour d'un générateur pulsé de type Blumlein.

\section{DISPOSITIF EXPÉRIMENTAL}

La source plasma est confinée dans un capillaire en polyacétal de diamètre intérieur $3,5 \mathrm{~mm}$. La longueur de la colonne de plasma est $54 \mathrm{~mm}$. Le rapport d'aspect de cette géométrie, supérieur à 15 , est favorable à une AES. La décharge est déclenchée par un commutateur de très faible inductance $(8 \mathrm{nH})$. La géométrie de ce commutateur détermine certains paramètres importants de la décharge, dont le temps de commutation qui est inférieur à 70 ns. L'énergie est emmagasinée dans deux condensateurs plans qui possèdent une inductance propre négligeable devant celle du tube capillaire. Ils sont constitués de deux plaques en résine époxy recouvertes d'une couche de cuivre de $100 \mu \mathrm{m}$ d'épaisseur. La capacité totale de la ligne Blumlein est de $90 \mathrm{nF}$. Cette ligne peut être chargée à des tensions allant jusqu'à $40 \mathrm{kV}$. L'énergie maximale emmagasinée dans la ligne de mise en forme d'impulsion est d'environ $70 \mathrm{~J}$. Dans ce type de circuit, on peut estimer que seule la moitié de cette énergie ( $35 \mathrm{~J}$ ) est disponible pour la décharge, l'autre moitié étant dissipée dans le plasma de commutation. La rapidité de la ligne (inductance totale de $20 \mathrm{nH}$, capacité de $45 \mathrm{nF}$ par condensateur) permet de produire, sur ce générateur, des courants pulsés de plus de $40 \mathrm{kA}$. Le taux de montée du courant, particulièrement important pour obtenir un effet de constriction magnétique selon l'axe du capillaire, atteint environ $1,8.10^{12}$ ampères par seconde. 
Le diagnostic du plasma est réalisé en visée longitudinale. Un spectromètre de type "Spartuvix" de faible résolution spectrale est placé dans le prolongement de l'axe du capillaire. Le domaine spectral couvert par ce diagnostic spectroscopique s'étend de 4,5 à $95 \mathrm{~nm}$, couvrant ainsi toute les longueurs d'onde de l'extrême ultraviolet. Il est équipé d'un réseau par transmission en or de périodicité 2000 traits par millimètre. Un miroir torique monté en avant du réseau le protège entre autres des débris. Les spectres sont enregistrés par un détecteur à galette microcanaux (MCP) couplé à une caméra intensifiée (ICCD). Différentes conductances sont introduites sur le trajet du faiceau. Elles sont destinées à provoquer des pertes de charge significatives entre la pression qui règne à l'intérieur du capillaire, de l'ordre du millibar, et celle de la chambre de détection, avoisinant $10^{-6}$ millibar, en sortie du spectromètre.

\section{SPECTROSCOPIE EUV}

Un spectre typique obtenu sur ce dispositif est présenté en figure 1. Il a été enregistré dans un plasma d'argon à une pression de 1 mbar. Ce spectre est présenté en comparaison avec celui mieux résolu de la référence [2]. Il fait apparaître très clairement le présence de raies d'argon 10 et 11 fois ionisés.

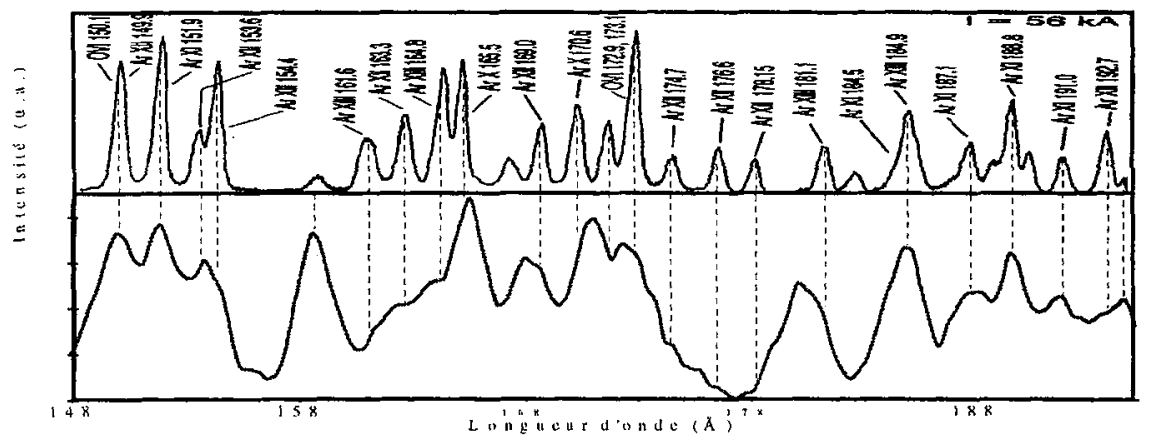

Figure 1: Spectre EUV d'un plasma d'argon à une pression de $1 \mathrm{mbar}$

La température électronique a été estimée entre 30 et $100 \mathrm{eV}$. Les calculs numériques ont montré que, dans de telles conditions expérimentales, le plasma avait fortement tendance à produire un "pinch", dont le rayon au plus fort de la compression pouvait être inférieur à $200 \mu \mathrm{m}$, et présentait une densité electronique de l'ordre de $10^{20} \mathrm{~cm}^{-3}$.

\section{Remerciements}

Ce travail a bénéficié du soutien financier du TMR européen FACADIX .

\section{Références}

1. D. Hong, R. Dussart, C. Cachoncinlle, W. Rosenfeld, S. Götze, J. Pons, R Viladrosa, C. Fleurier, J.M. Pouvesle, Rev. Sci. Instrum. 71, 15, (2000).

2. J.J. Rocca, O.D. Cortazar, D. Szapiro, K. Floyd, F.G. Tomasel, Phys. Rev. E. 47, 1299, (1993) 